\title{
The impact of acetylation and deacetylation on the p53 pathway
}

\author{
Christopher L. Brooks ${ }^{1 凶}$, Wei Gu ${ }^{2}$ \\ ${ }^{1}$ Stemline Therapeutics, Inc., New York, NY 10128, USA \\ 2 Institute for Cancer Genetics, and Department of Pathology College of Physicians \& Surgeons, Columbia University, New York, \\ NY 10032, USA \\ $\triangle$ Correspondence: cbrooks@stemline.com \\ Received May 5, 2011 Accepted May 9, 2011
}

\begin{abstract}
The p53 tumor suppressor is a sequence-specific transcription factor that undergoes an abundance of post-translational modifications for its regulation and activation. Acetylation of p53 is an important reversible enzymatic process that occurs in response to DNA damage and genotoxic stress and is indispensible for p53 transcriptional activity. p53 was the first non-histone protein shown to be acetylated by histone acetyl transferases, and a number of more recent in vivo models have underscored the importance of this type of modification for p53 activity. Here, we review the current knowledge and recent findings of p53 acetylation and deacetylation and discuss the implications of these processes for the p53 pathway.
\end{abstract}

KEYWORDS p53, Mdm2, acetylation, deacetylation, destabilization, ubiquitination, transcriptional activation and stability

\section{INTRODUCTION}

The tumor suppressor p53 is an essential and key transcription factor in cellular stress response pathways (Levine and Oren, 2009). The abundance of upstream regulators underscores the critical importance of keeping this protein under tight control. Under normal, non-stressed physiological conditions, p53 protein is maintained at low levels within a cell by its predominant negative regulator Mdm2. However, during times of genotoxic and other cellular stresses, a number of mechanisms allow for the quick accumulation and activation of $\mathrm{p} 53$ in order to prevent uncontrolled growth of cells harboring deleterious genetic alterations.

A number of post-translational modifications can occur in p53 that have critical effects on its stability and function, including phosphorylation, acetylation, sumoylation, neddylation, and methylation (Kruse and Gu, 2009a). While many if not all of these modifications have been described in in vitro and cell culture systems, more recent animal studies have been conducted to assess the true physiological function of a number of these modifications (Feng et al., 2005; Krummel et al., 2005). The picture that has emerged is a highly intricate and complex combination of events that ultimately lead to p53-dependent cell growth arrest or apoptosis, and key posttranslational modifications are imperative for these events to occur.

Post-translational acetylation, or the reversible process by which acetyl groups are enzymatically placed onto the $\varepsilon$ amino group of lysine residues of target proteins, is a well studied event that occurs in histone tails during transcription (MacDonald and Howe, 2009). Histone acetylation is an enzymatic event that has been shown to occur both globally throughout the genome as well as at specific promoters and is an indicator of actively transcribed genes (Harbison et al., 2004; Wang et al., 2008b). The first non-histone substrate shown to be acetylated by histone acetyl transferases (HATs) was p53, which was a novel and previously unknown mechanism for protein activation (Gu and Roeder, 1997). Since this seminal characterization, other non-histone proteins have been shown to be acetylated that are involved in diverse cellular processes such as the cell cycle, gene splicing, and nuclear transport (Kim et al., 2006; Choudhary et al., 2009). Recent descriptions of the in vivo acetylome have not only identified lysine acetylation sites in upwards of 1700 proteins, but they have also shown that several regulators of the p53 pathway are also acetylated, including Daxx, PML, PTEN, and HAUSP (Choudhary et al., 2009). These findings suggest that acetylation plays a key role in the regulation and activity of virtually all nuclear processes and many 


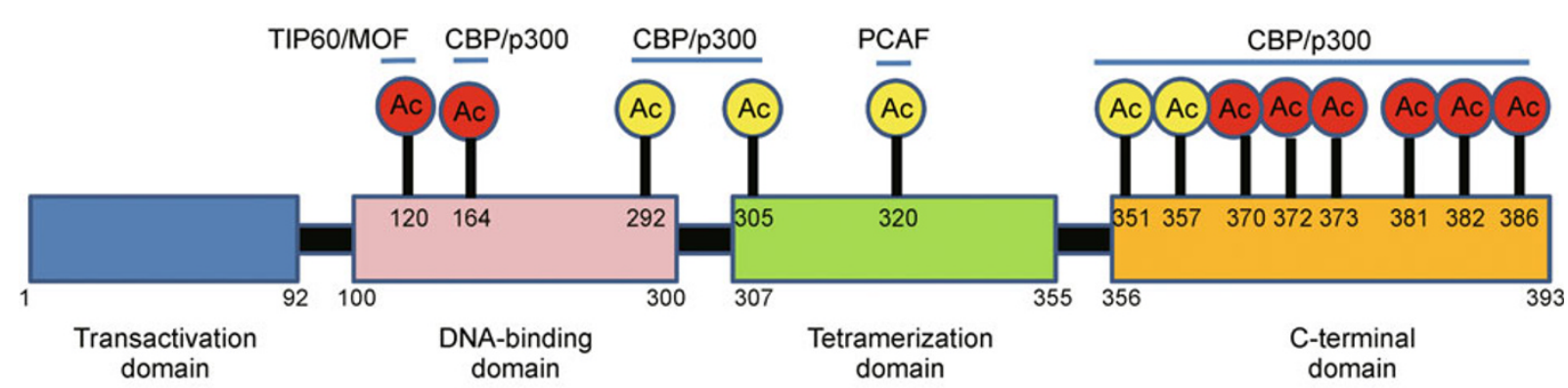

Figure 1. Schematic diagram of p53 functional domains and overview of p53 acetylation sites. The eight acetylation sites that are indispensible for p53 activation are indicated in red. The corresponding major modifying enzymes are also indicated.

cytoplasmic functions (Choudhary et al., 2009). In this review, we highlight the important effect that acetylation has on p53 function and regulation and how this type of modification may have significant implications for the signaling pathways that are induced in response to stress.

\section{ACETYLATION OF P53}

p53 was the first non-histone protein shown to be acetylated by HATs (Gu and Roeder, 1997). Enhancement of p53 acetylation levels strongly correlates with protein stabilization and activation in response to cellular stress (Luo et al., 2000; Rodriguez et al., 2000; Barlev et al., 2001; Ito et al., 2001; Knights et al., 2006; Li et al., 2007; Kim et al., 2008; Zhao et al., 2008). Moreover, acetylation of p53 was found to stimulate its sequence-specific DNA-binding (Gu and Roeder, 1997; Sakaguchi et al., 1998; Liu et al., 1999; Luo et al., 2004; Zhao et al., 2006). C-terminal lysine residues at positions 164 , $305,370,372,373,381$, and 382 have been shown to be acetylated by the HAT p300/CREB-binding protein (CBP) and lysine 320 by the HAT p300/CBP-associated factor (PCAF) (Fig. 1) (Gu and Roeder, 1997; Sakaguchi et al., 1998; Liu et al., 1999; Wang et al., 2003). Importantly, mutations in the p300/CBP gene are frequently found in a number of cancers (Goodman and Smolik, 2000; lyer et al., 2004). Acetylation of these sites is also important as a docking site for the subsequent recruitment of HAT and other transcription cofactors to promoter regions, and p300/CBP can enhance histone acetylation and promote transcription in the vicinity of target genes (Goodman and Smolik, 2000).

More recently, two other acetyl transferases from the MYST family, hMOF and TIP60, were shown to acetylate p53 at position 120, which is located within the DNA binding domain (Sykes et al., 2006; Tang et al., 2006). These findings showed that p53 was acetylated at a unique lysine residue outside of the C-terminal domain. This lysine residue is also the only site of modification in p53 that is evolutionarily conserved and is a recurrent site of mutation in several human cancers, suggesting its important role in controlling p53 function. Interestingly, modification of this site has no effect on DNA binding or protein stability; however it was shown to be critical for the activation of the proapoptotic genes PUMA and BAX (Sykes et al., 2006; Tang et al., 2006). The HATs hMOF and TIP60 were also shown to be recruited to sites of transcription by $\mathrm{p} 53$ and were able to acetylate both p53 and histones. Importantly, K120 acetylation is induced after DNA damage and after activation of p19ARF by oncogenic stress (Tang et al., 2006; Mellert et al., 2007). Interestingly, mutation of this lysine residue to an arginine (K120R) abrogated p53-mediated apoptosis but not cell growth arrest (Tang et al., 2006). These data suggest that acetylation of lysine 120 is crucial for the differential activation of p53 target genes. The importance of this modification as well as other types of regulation for the mechanism by which p53 modulates cell cycle arrest and apoptosis are discussed below.

The dependence of the acetylation-dependent activity of p53 in vivo on these sites of modification seemed to be cell type specific, as the C-terminal acetylation-deficient p53-6KR knockin mouse, where the six C-terminal lysine residues subject to acetylation were substituted with arginine, had reduced p53-dependent gene expression after DNA damage in both thymocytes and embryonic stem cells (Feng et al., 2005). Significant differences were not seen in the embryonic fibroblasts from these mice. Moreover, a p53-7KR knockin mouse containing mutations at seven $\mathrm{C}$-terminal acetylation sites analogous to the human p53-6KR mutant showed no defects in p53-mediated cell growth arrest and apoptosis (Krummel et al., 2005). The in vivo data suggested that these specific sites of acetylation may only have modest effects on overall growth arrest and apoptotic control, though some defects were observed in specific cell types. Moreover, a knockin mutation introduced at the PCAF acetylation site Lys320 also showed no defects in the expression of p53 target genes, and in fact showed an increase in the expression of proapoptotic genes NOXA, PUMA, APAF1, and PIDD in mouse embryonic fibroblasts (Chao et al., 2006). This study also demonstrated an increase in apoptosis of specific cell types from these mice after irradiation, including thymocytes, epithelial cells of the small intestine, and retinal cells (Chao et al., 2006). Though the mechanism behind these observations remains unclear, the lack of significant 
defects in p53-dependent gene expression in these in vivo models suggests a more complex mode that may include other acetylation sites within the molecule.

Recently, a previously undiscovered acetylation site mediated by p300/CBP was described for p53 (Tang et al., 2008). The previously unfound lysine residue at position 164 was identified by a mass spectrometric analysis of all acetylation sites in p53. Interestingly, deletion of this site in combination with other known acetylation sites in p53 (p53$8 \mathrm{KR}$ ) completely abrogated p53-dependent transactivation of p21 and the ability to induce cell growth arrest. Importantly, single acetylation defects at any of these sites individually had no effect on the transactivation ability of p53, suggesting that the acetylation sites described in vivo for the p53-6KR and p53-7KR knockin mice can be compensated for by acetylation at other sites. These findings not only confirm previous in vivo studies showing p53-dependent gene expression in p53 acetylation deficient mice, but also show that acetylation is indispensible for p53 activation. It will be interesting to see what in vivo effects the alterations of these sites have in animal models.

\section{UBIQUITINATION AND ACETYLATION}

The function and activity of p53 is highly regulated by acetylation, but an important step in the response to DNA damage is the rapid and efficient stabilization of p53 protein levels. The physiological levels of p53 are tightly regulated by the $26 \mathrm{~S}$ proteosome pathway and ubiquitination, which is a mutually exclusive modification that occurs on the same lysine residues used by acetyltransferases. The predominant E3 ubiquitin ligase for p53 is the RING finger domain containing protein Mdm2, and loss of this gene causes p53dependent embryonic lethality at day E6.5 (Jones et al., 1995; Montes de Oca Luna et al., 1995). Under normal physiological conditions, Mdm2-mediated ubiquitination targets p53 to the $26 \mathrm{~S}$ proteosome for degradation, thereby maintaining low protein levels within the cell. However, upon a number of cellular stress conditions, p53 protein levels need to be quickly stabilized for activation, and this process occurs through several mechanisms, including post-translational modifications, physical sequestration, and degradation of the components of the p53 pathway (Brooks and $\mathrm{Gu}, 2006$ ). For example, DNA damage-induced phosphorylation of p53 by the kinases ATM, ATR, Chk1, and Chk 2 at serine 15 and 20 leads to a disruption of the p53-Mdm2 interaction (Shieh et al., 1997; Appella and Anderson, 2001; Kruse and Gu, 2009a). Although subsequent in vivo knockin models of these modification sites showed no defects in stress-induced p53 stabilization, the combinatorial mutation of these sites showed a more pronounced defect in p53 stabilization (Chao et al., 2006).

Mdm2 is also a direct target of several upstream regulators for efficient stabilization of p53. The tumor suppressor ARF has been shown to indirectly stabilize p53 by interacting with Mdm2 in response to oncogenic stress (Sherr, 2001; Sharpless and DePinho, 2004). ARF can induce p53dependent cell growth arrest or apoptosis by binding to and sequestering Mdm2 in the nucleolus, thereby stabilizing nucleoplasmic p53. ARF also directly inhibits the enzymatic activity of Mdm2 and may have effects on another p53 E3 ligase, ARF-BP1 (Chen et al., 2005; Zhong et al., 2005). Acetylation of Mdm2 within the RING domain by $\mathrm{p} 300 / \mathrm{CBP}$ has also been shown to disrupt the p53-Mdm2 interaction and promote p53 stabilization (Wang et al., 2004). Conversely, Mdm2 can inhibit both p300/CBP and PCAF-mediated acetylation of p53 by suppressing the acetyltransferase activity and by recruiting the histone deacetylase HDAC1 to p53 (Benkirane et al., 2010). Mdm2 has also been shown to ubiquitinate Tip60 and PCAF, both of which acetylate p53. Together, these data illustrate that the abundance of mechanisms available underlying the disruption of the p53Mdm2 interaction augments the negative regulation impact Mdm2 has on p53. Moreover, both acetylation and ubiquitination occur in the same lysine residues, showing a complex network of regulators for p53 activity, stability, and activation. Indeed, acetylation of p53 was shown to inhibit Mdm2mediated degradation in vitro and in cell culture ( $\mathrm{Li}$ et al., 2002).

The p53-dependent embryonic lethality of the Mdm2 knockout mouse reveals the importance of this protein in the p53 pathway; however more recently it has been shown that p53 is still efficiently degraded in the absence of Mdm2 in cells from Mdm2 null mice (Ringshausen et al., 2006). These results suggest that alternative mechanisms for p53 degradation exist within cells that are independent of Mdm2. Indeed, several other ubiquitin ligases have been described for $\mathrm{p} 53$ in vitro and in cell culture, including ARF-BP1/Mule, COP1, Pirh2, MSL2, p300/CBP, and E4F1 (Grossman et al., 2003; Leng et al., 2003; Dornan et al., 2004; Chen et al., 2005; Kruse and $\mathrm{Gu}, 2009 \mathrm{~b}$ ). Ubiquitination of $\mathrm{p} 53$ by these proteins has been shown to either cause the degradation of p53 or lead to a change in subcellular localization. Interestingly, p300/CBP possesses both acetyltransferase and ubiquitinase activity for $\mathrm{p} 53$. Although this protein does not possess an obvious ubiqutin ligase domain (RING or HECT), it was shown to act as an E4 ubiquitin ligase for p53 and polyubiqutinate p53 (Shi et al., 2009). In this regard, p300/ CBP works in conjunction with Mdm2 to efficiently polyubiquitinate $\mathrm{p} 53$, which is a key signaling mechanism for recognition by the $26 \mathrm{~S}$ proteosome. Although the p300/CBPMdm2 interaction has been shown to be important for Mdm2 activity on p53 in vitro, further in vivo studies will be able to dissect these interactions and their combination effects on p53.

In addition to acetyltransferase activity, PCAF was also recently shown to have intrinsic ubiquitination activity on lysine 320 of p53 (Benkirane et al., 2010). Moreover, PCAF 


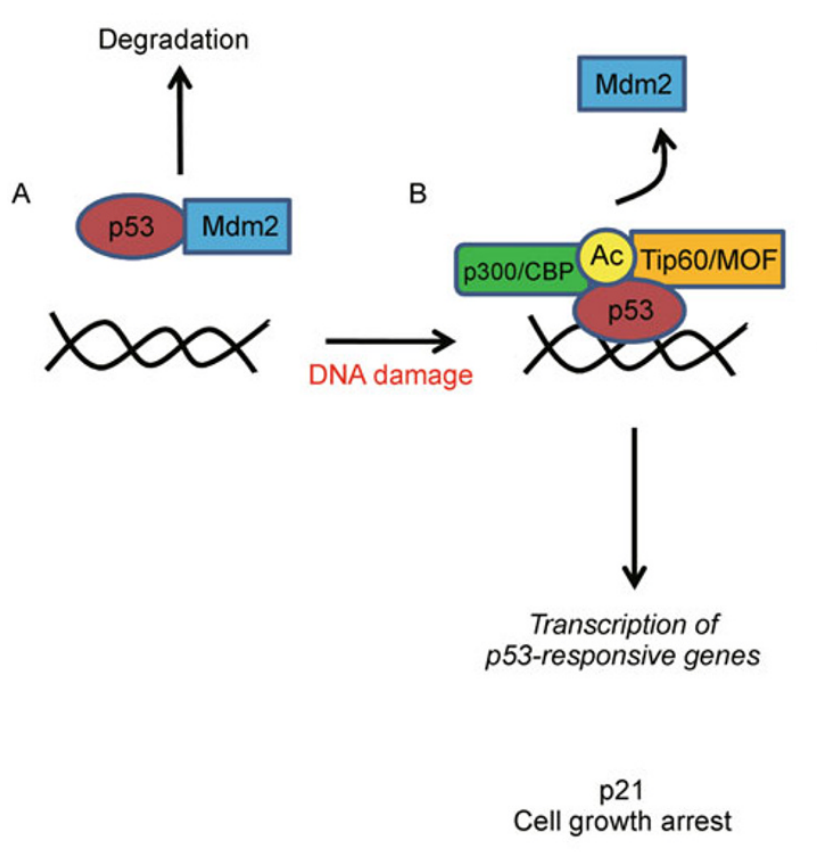

C
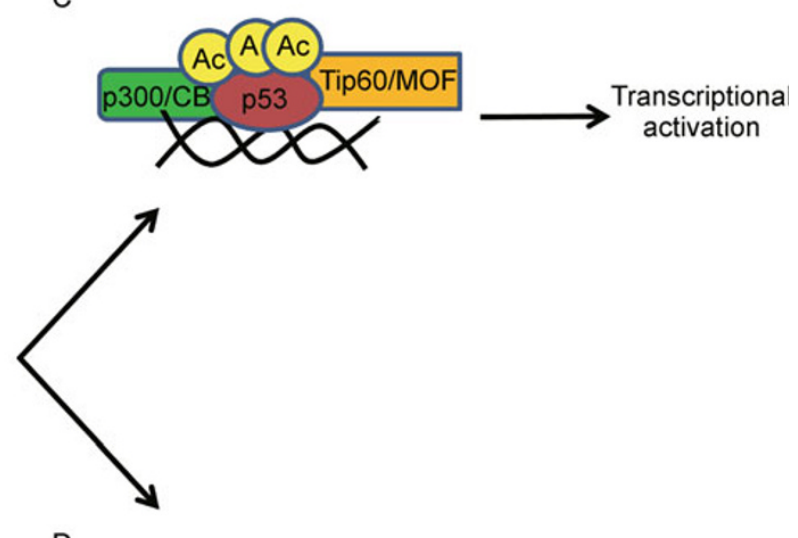

D
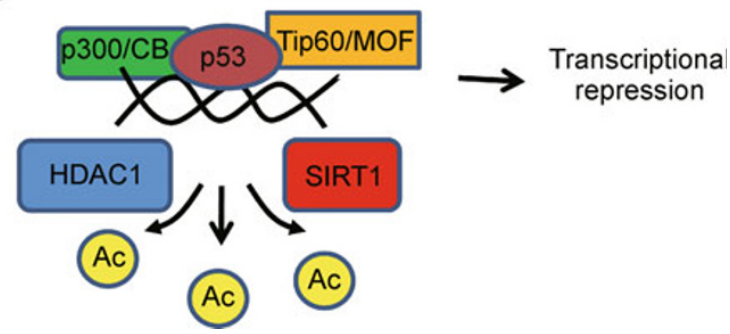

Figure 2. The role of p53 acetylation in gene regulation. (A) Unacetylated p53 is capable of activating genes that are involved in the negative regulation of $\mathrm{p} 53$, such as Mdm2, as a mechanism to keep p53 protein levels low during times of normal homeostasis. (B) Upon DNA damage, acetylation of p53 allows for the disruption of the Mdm2-p53 interaction and the recruitment of specific HATs to the promoters of genes involved in DNA repair and cell cycle control. (C) Full acetylation of p53 at all key acetylation sites promotes the activation of proapoptotic genes. (D) SIRT1 and HDAC1 are deacetylases that can reverse p53 acetylation and lead to transcriptional repression.

can directly ubiquitinate Mdm2, leading to PCAF-mediated degradation of the protein. Mdm2 also has auto-ubiquitinase activity and can self-ubiquitinate, and PCAF-mediated degradation of Mdm2 suggests an interesting alternative mechanism for Mdm2 degradation. Importantly, an Mdm2 RINGdeficient knockin mouse model showed comparable Mdm2 protein levels with the wild-type, suggesting that alternative mechanisms exist to control Mdm2 protein levels (Itahana et al., 2007). Moreover, the newly found enzymatic activity of PCAF shows that this protein can promote p53 stability and activation by both degrading Mdm2 and activating p53.

\section{DEACETYLATION OF P53}

The critical effects of acetylation on p53 activity show that this type of modification has to be tightly regulated and reversible. Indeed, mammalian histone deacetylases (HDACs) downregulate p53 transcriptional activity, and p53 acetylation can be antagonized by specific HDAC protein complexes (Kruse and $\mathrm{Gu}, 2009 \mathrm{a}$ ). HDACs are categorized based on their similarities to yeast homologues and are grouped into class I, II, and III (de Ruijter et al., 2003). p53 acetylation can be reversed by a protein complex containing HDAC1 (Luo et al., 2000). Interaction with this complex reduces the steady-state levels of acetylated p53 and inhibits p53-dependent transcriptional activation, cell growth arrest, and apoptosis (Fig. 2). In addition, the class III NAD +-dependent deacetylase SIRT1 was shown to deacetylate p53 (Luo et al., 2001; Vaziri et al., 2001). SIRT1-mediated deacetylation of p53 inactivates the sequence-specific transcriptional activity of the protein and represses p53-mediated cell growth arrest and apoptosis in response to DNA damage and oxidative stress. This process also prevented p53-dependent transactivation of p21 (Luo et al., 2001).

The role of SIRT1 and deacetylation in the p53 pathway is complex. SIRT1 has roles in diverse signaling pathways from metabolism to cellular senescence and aging (Bordone and Guarente, 2005). Although SIRT1 has a direct negative effect on p53 transcriptional activity, embryonic fibroblasts from a Sir2a knockout mouse model have exhibited impaired p53 functions (Wang et al., 2008a). Despite the presence of hyperacetylated p53 in these mice, these cells had an increased capacity to proliferate in response to chronic, sub-lethal doses of oxidative stress and failed to mount an adequate DNA damage response (Cheng et al., 2003; Chua et al., 2005). Moreover, SIRT1 has been shown to relocalize to sites of DNA double stranded breaks to help promote DNA repair and can deacetylate the DNA repair protein NBS1 
(Yuan et al., 2007), WRN (Li et al., 2008), and XPA (Fan and Luo, 2010). Embryonic fibroblasts from Sirt1-null mice show defects in DNA repair, despite a high steady-state level of acetylated $\mathrm{p} 53$. These findings, together with the seemingly opposing results of p53 regulation, suggest that SIRT1 and deacetylation may have an important role in p53-mediated cellular senescence. It is well established that SIRT1 has critical functions in longevity and aging in other organisms such as yeast (Bordone and Guarente, 2005). SIRT1mediated deacetylation of p53 in response to low levels of DNA damage may therefore promote cellular senescence and help cellular recovery from non-lethal genotoxic events (Wang et al., 2008a). This hypothesis may be complicated by other as yet undefined regulators in this pathway or other mechanisms for balancing the ratio or levels of acetylated p53, since SIRT1 does not fit the classical model of a true tumor suppressor and has not been found mutated in human cancers (Luo et al., 2001; Huang et al., 2008). Nevertheless, p53 has a clear functional role in cellular senescence and premature aging (Tyner et al., 2002), and SIRT1-mediated deacetylation may promote this phenotype and provide a regulatory link to cellular longevity.

\section{CONCLUSIONS}

Our understanding of the intricate regulatory control of p53 function continues to evolve. It has become clear from mouse knockin studies that p53 requires a multitude of posttranslational modifications as well as additional adaptations to become fully activated. Moreover, the precise combination of modifications may act as a signal to trigger specific pathways. Acetylation plays a critical role in the functional activity of p53 and is indispensible for transcriptional activity. Since several p53 coactivators, such as ASPPs and 53BP1, are known to bind to this region, the identification of acetylation sites outside the C-terminal domain raises the possibility that modification of these lysine residues may promote the activation of different p53 target genes (Joo et al., 2002; Li et al., 2007; Sullivan and Lu, 2007). Further in vivo investigation of these acetylation sites and the impact they have on p53 function is critical for a full understanding of these regulatory processes.

\section{REFERENCES}

Appella, E., and Anderson, C.W. (2001). Post-translational modifications and activation of p53 by genotoxic stresses. Eur J Biochem 268, 2764-2772.

Barlev, N.A., Liu, L., Chehab, N.H., Mansfield, K., Harris, K.G., Halazonetis, T.D., and Berger, S.L. (2001). Acetylation of p53 activates transcription through recruitment of coactivators/histone acetyltransferases. Mol Cell 8, 1243-1254.

Benkirane, M., Sardet, C., and Coux, O. (2010). Lessons from interconnected ubiquitylation and acetylation of p53: think metastable networks. Biochem Soc Trans 38, 98-103.
Bordone, L., and Guarente, L. (2005). Calorie restriction, SIRT1 and metabolism: understanding longevity. Nat Rev Mol Cell Biol 6, 298-305.

Brooks, C.L., and Gu, W. (2006). p53 ubiquitination: Mdm2 and beyond. Mol Cell 21, 307-315.

Chao, C., Wu, Z., Mazur, S.J., Borges, H., Rossi, M., Lin, T., Wang, J. Y., Anderson, C.W., Appella, E., and Xu, Y. (2006). Acetylation of mouse p53 at lysine 317 negatively regulates p53 apoptotic activities after DNA damage. Mol Cell Biol 26, 6859-6869.

Chen, D., Kon, N., Li, M., Zhang, W., Qin, J., and Gu, W. (2005). ARF$\mathrm{BP} 1 /$ Mule is a critical mediator of the ARF tumor suppressor. Cell 121, 1071-1083.

Cheng, H.L., Mostoslavsky, R., Saito, S., Manis, J.P., Gu, Y., Patel, P., Bronson, R., Appella, E., Alt, F.W., and Chua, K.F. (2003). Developmental defects and p53 hyperacetylation in Sir2 homolog (SIRT1)-deficient mice. Proc Natl Acad Sci U S A 100, 10794-10799.

Choudhary, C., Kumar, C., Gnad, F., Nielsen, M.L., Rehman, M., Walther, T.C., Olsen, J.V., and Mann, M. (2009). Lysine acetylation targets protein complexes and co-regulates major cellular functions. Science 325, 834-840.

Chua, K.F., Mostoslavsky, R., Lombard, D.B., Pang, W.W., Saito, S., Franco, S., Kaushal, D., Cheng, H.L., Fischer, M.R., Stokes, N., et al. (2005). Mammalian SIRT1 limits replicative life span in response to chronic genotoxic stress. Cell Metab 2, 67-76.

de Ruijter, A.J., van Gennip, A.H., Caron, H.N., Kemp, S., and van Kuilenburg, A.B. (2003). Histone deacetylases (HDACs): characterization of the classical HDAC family. Biochem $\mathrm{J} 370$, 737-749.

Dornan, D., Wertz, I., Shimizu, H., Arnott, D., Frantz, G.D., Dowd, P., O'Rourke, K., Koeppen, H., and Dixit, V.M. (2004). The ubiquitin ligase COP1 is a critical negative regulator of p53. Nature 429, 86-92.

Fan, W., and Luo, J. (2010). SIRT1 regulates UV-induced DNA repair through deacetylating XPA. Mol Cell 39, 247-258.

Feng, L., Lin, T., Uranishi, H., Gu, W., and Xu, Y. (2005). Functional analysis of the roles of posttranslational modifications at the p53 C terminus in regulating p53 stability and activity. Mol Cell Biol 25 , 5389-5395.

Goodman, R.H., and Smolik, S. (2000). CBP/p300 in cell growth, transformation, and development. Genes Dev 14, 1553-1577.

Grossman, S.R., Deato, M.E., Brignone, C., Chan, H.M., Kung, A.L., Tagami, H., Nakatani, Y., and Livingston, D.M. (2003). Polyubiquitination of $p 53$ by a ubiquitin ligase activity of p300. Science 300 , 342-344.

Gu, W., and Roeder, R.G. (1997). Activation of p53 sequence-specific DNA binding by acetylation of the p53 C-terminal domain. Cell 90, 595-606.

Harbison, C.T., Gordon, D.B., Lee, T.I., Rinaldi, N.J., Macisaac, K.D., Danford, T.W., Hannett, N.M., Tagne, J.B., Reynolds, D.B., Yoo, J., et al. (2004). Transcriptional regulatory code of a eukaryotic genome. Nature 431, 99-104.

Huang, J., Gan, Q., Han, L., Li, J., Zhang, H., Sun, Y., Zhang, Z., and Tong, T. (2008). SIRT1 overexpression antagonizes cellular senescence with activated ERK/S6k1 signaling in human diploid fibroblasts. PLoS One 3, e1710.

Itahana, K., Mao, H., Jin, A., Itahana, Y., Clegg, H.V., Lindström, M.S., Bhat, K.P., Godfrey, V.L., Evan, G.I., and Zhang, Y. (2007). 
Targeted inactivation of Mdm2 RING finger E3 ubiquitin ligase activity in the mouse reveals mechanistic insights into p53 regulation. Cancer Cell 12, 355-366.

Ito, A., Lai, C.H., Zhao, X., Saito, S., Hamilton, M.H., Appella, E., and Yao, T.P. (2001). p300/CBP-mediated p53 acetylation is commonly induced by $\mathrm{p} 53$-activating agents and inhibited by MDM2. EMBO J 20, 1331-1340.

lyer, N.G., Ozdag, H., and Caldas, C. (2004). p300/CBP and cancer. Oncogene 23, 4225-4231.

Jones, S.N., Roe, A.E., Donehower, L.A., and Bradley, A. (1995). Rescue of embryonic lethality in Mdm2-deficient mice by absence of p53. Nature 378, 206-208.

Joo, W.S., Jeffrey, P.D., Cantor, S.B., Finnin, M.S., Livingston, D.M., and Pavletich, N.P. (2002). Structure of the 53BP1 BRCT region bound to p53 and its comparison to the Brca1 BRCT structure. Genes Dev 16, 583-593.

Kim, J.E., Chen, J., and Lou, Z. (2008). DBC1 is a negative regulator of SIRT1. Nature 451, 583-586.

Kim, S.C., Sprung, R., Chen, Y., Xu, Y., Ball, H., Pei, J., Cheng, T., Kho, Y., Xiao, H., Xiao, L., et al. (2006). Substrate and functional diversity of lysine acetylation revealed by a proteomics survey. Mol Cell 23, 607-618.

Knights, C.D., Catania, J., Di Giovanni, S., Muratoglu, S., Perez, R., Swartzbeck, A., Quong, A.A., Zhang, X., Beerman, T., Pestell, R. G., et al. (2006). Distinct p53 acetylation cassettes differentially influence gene-expression patterns and cell fate. J Cell Biol 173, 533-544.

Krummel, K.A., Lee, C.J., Toledo, F., and Wahl, G.M. (2005). The Cterminal lysines fine-tune P53 stress responses in a mouse model but are not required for stability control or transactivation. Proc Natl Acad Sci U S A 102, 10188-10193.

Kruse, J.P., and Gu, W. (2009a). Modes of p53 regulation. Cell 137, 609-622.

Kruse, J.P., and Gu, W. (2009b). MSL2 promotes Mdm2-independent cytoplasmic localization of p53. J Biol Chem 284, 3250-3263.

Leng, R.P., Lin, Y., Ma, W., Wu, H., Lemmers, B., Chung, S., Parant, J. M., Lozano, G., Hakem, R., and Benchimol, S. (2003). Pirh2, a p53-induced ubiquitin-protein ligase, promotes p53 degradation. Cell 112, 779-791.

Levine, A.J., and Oren, M. (2009). The first 30 years of p53: growing ever more complex. Nat Rev Cancer 9, 749-758.

Li, A.G., Piluso, L.G., Cai, X., Gadd, B.J., Ladurner, A.G., and Liu, X. (2007). An acetylation switch in p53 mediates holo-TFIID recruitment. Mol Cell 28, 408-421.

Li, K., Casta, A., Wang, R., Lozada, E., Fan, W., Kane, S., Ge, Q., Gu, W., Orren, D., and Luo, J. (2008). Regulation of WRN protein cellular localization and enzymatic activities by SIRT1-mediated deacetylation. J Biol Chem 283, 7590-7598.

Li, M., Luo, J., Brooks, C.L., and Gu, W. (2002). Acetylation of p53 inhibits its ubiquitination by Mdm2. J Biol Chem 277, 50607-50611.

Liu, L., Scolnick, D.M., Trievel, R.C., Zhang, H.B., Marmorstein, R., Halazonetis, T.D., and Berger, S.L. (1999). p53 sites acetylated in vitro by PCAF and p300 are acetylated in vivo in response to DNA damage. Mol Cell Biol 19, 1202-1209.

Luo, J., Li, M., Tang, Y., Laszkowska, M., Roeder, R.G., and Gu, W. (2004). Acetylation of p53 augments its site-specific DNA binding both in vitro and in vivo. Proc Natl Acad Sci U S A 101, 2259-2264.

Luo, J., Nikolaev, A.Y., Imai, S., Chen, D., Su, F., Shiloh, A., Guarente,
L., and Gu, W. (2001). Negative control of p53 by Sir2alpha promotes cell survival under stress. Cell 107, 137-148.

Luo, J., Su, F., Chen, D., Shiloh, A., and Gu, W. (2000). Deacetylation of p53 modulates its effect on cell growth and apoptosis. Nature 408, 377-381.

MacDonald, V.E., and Howe, L.J. (2009). Histone acetylation: where to go and how to get there. Epigenetics 4, 139-143.

Mellert, H., Sykes, S.M., Murphy, M.E., and McMahon, S.B. (2007). The ARF/oncogene pathway activates p53 acetylation within the DNA binding domain. Cell Cycle 6, 1304-1306.

Montes de Oca Luna, R., Wagner, D.S., and Lozano, G. (1995). Rescue of early embryonic lethality in mdm2-deficient mice by deletion of p53. Nature 378, 203-206.

Ringshausen, I., O'Shea, C.C., Finch, A.J., Swigart, L.B., and Evan, G.I. (2006). Mdm2 is critically and continuously required to suppress lethal p53 activity in vivo. Cancer Cell 10, 501-514.

Rodriguez, M.S., Desterro, J.M., Lain, S., Lane, D.P., and Hay, R.T. (2000). Multiple C-terminal lysine residues target p53 for ubiquitinproteasome-mediated degradation. Mol Cell Biol 20, 8458-8467.

Sakaguchi, K., Herrera, J.E., Saito, S., Miki, T., Bustin, M., Vassilev, A., Anderson, C.W., and Appella, E. (1998). DNA damage activates p53 through a phosphorylation-acetylation cascade. Genes Dev 12, 2831-2841.

Sharpless, N.E., and DePinho, R.A. (2004). Telomeres, stem cells, senescence, and cancer. J Clin Invest 113, 160-168.

Sherr, C.J. (2001). The INK4a/ARF network in tumour suppression. Nat Rev Mol Cell Biol 2, 731-737.

Shi, D., Pop, M.S., Kulikov, R., Love, I.M., Kung, A.L., and Grossman, S.R. (2009). CBP and p300 are cytoplasmic E4 polyubiquitin ligases for p53. Proc Natl Acad Sci U S A 106, 16275-16280.

Shieh, S.Y., Ikeda, M., Taya, Y., and Prives, C. (1997). DNA damageinduced phosphorylation of p53 alleviates inhibition by MDM2. Cell 91, 325-334.

Sullivan, A., and Lu, X. (2007). ASPP: a new family of oncogenes and tumour suppressor genes. Br J Cancer 96, 196-200.

Sykes, S.M., Mellert, H.S., Holbert, M.A., Li, K., Marmorstein, R., Lane, W.S., and McMahon, S.B. (2006). Acetylation of the p53 DNA-binding domain regulates apoptosis induction. Mol Cell 24, 841-851.

Tang, Y., Luo, J., Zhang, W., and Gu, W. (2006). Tip60-dependent acetylation of $\mathrm{p} 53$ modulates the decision between cell-cycle arrest and apoptosis. Mol Cell 24, 827-839.

Tang, Y., Zhao, W., Chen, Y., Zhao, Y., and Gu, W. (2008). Acetylation is indispensable for p53 activation. Cell 133, 612-626.

Tyner, S.D., Venkatachalam, S., Choi, J., Jones, S., Ghebranious, N., Igelmann, H., Lu, X., Soron, G., Cooper, B., Brayton, C., et al. (2002). p53 mutant mice that display early ageing-associated phenotypes. Nature 415, 45-53.

Vaziri, H., Dessain, S.K., Ng Eaton, E., Imai, S.I., Frye, R.A., Pandita, T.K., Guarente, L., and Weinberg, R.A. (2001). hSIR2(SIRT1) functions as an NAD-dependent p53 deacetylase. Cell 107, 149-159.

Wang, R.H., Sengupta, K., Li, C., Kim, H.S., Cao, L., Xiao, C., Kim, S., Xu, X., Zheng, Y., Chilton, B., et al. (2008a). Impaired DNA damage response, genome instability, and tumorigenesis in SIRT1 mutant mice. Cancer Cell 14, 312-323.

Wang, X., Taplick, J., Geva, N., and Oren, M. (2004). Inhibition of p53 degradation by Mdm2 acetylation. FEBS Lett 561, 195-201. 
Wang, Y.H., Tsay, Y.G., Tan, B.C., Lo, W.Y., and Lee, S.C. (2003). Identification and characterization of a novel p300-mediated p53 acetylation site, lysine 305. J Biol Chem 278, 25568-25576.

Wang, Z., Zang, C., Rosenfeld, J.A., Schones, D.E., Barski, A., Cuddapah, S., Cui, K., Roh, T.Y., Peng, W., Zhang, M.Q., et al. (2008b). Combinatorial patterns of histone acetylations and methylations in the human genome. Nat Genet 40, 897-903.

Yuan, Z., Zhang, X., Sengupta, N., Lane, W.S., and Seto, E. (2007). SIRT1 regulates the function of the Nijmegen breakage syndrome protein. Mol Cell 27, 149-162.
Zhao, W., Kruse, J.P., Tang, Y., Jung, S.Y., Qin, J., and Gu, W. (2008). Negative regulation of the deacetylase SIRT1 by DBC1. Nature 451, 587-590.

Zhao, Y., Lu, S., Wu, L., Chai, G., Wang, H., Chen, Y., Sun, J., Yu, Y., Zhou, W., Zheng, Q., et al. (2006). Acetylation of p53 at lysine 373/ 382 by the histone deacetylase inhibitor depsipeptide induces expression of p21(Waf1/Cip1). Mol Cell Biol 26, 2782-2790.

Zhong, Q., Gao, W., Du, F., and Wang, X. (2005). Mule/ARF-BP1, a $\mathrm{BH} 3-$ only $\mathrm{E} 3$ ubiquitin ligase, catalyzes the polyubiquitination of Mcl-1 and regulates apoptosis. Cell 121, 1085-1095. 